\title{
Editorial introduction: BJET special Issue on critical success factors in institutional change Hugh Davis and Susan Eales
}

In the UK, starting in 2002, the Joint Information Systems Committee (JISC) began two influential funding programmes. One was the Exchange for Learning (X4L) Programme, which aimed to increase the practice of re-purposing of learning objects and content for educational purposes. The other was the Digital Libraries in the Classroom Programme which aimed to integrate currently available digital resources into core undergraduate teaching.

At the heart of both of these programmes was the intent to help UK institutions to build capacity to manage change, to acquire the skills needed to make informed decisions about adopting digital content within their core programmes and to understand the impact of this integration on student achievement, retention, recruitment and on institutional structures and practices. These were indeed highly ambitious programmes, and as they come to the end of their funding it seems appropriate to reflect on the achievement of these projects and others like them.

In 2006 JISC helped fund a workshop on "Critical Success Factors for Institutional Change" (CSFIC '06) which was co-located with the European Conference on Digital Libraries (ECDL '06). The papers in this special issue have emerged from this workshop. The intention is to present a range of experience papers which explain the issues that these projects have faced in making real and permanent change happen within their curricula. The papers are not intended to respond to the research agenda of any particular domain, but rather the authors were encouraged to tell the story as it happened, in language that should be clear and unambiguous to University managers and practitioners from all disciplines.

The seven papers presented here represent a range of projects, and present the problems of embedding change from number of different view points.

Three of the papers (Breslin et al.) (Davis and Fill) (Donald and Wallace) discuss the problems of embedding funded projects from an institutional management point of view. Three of the papers discuss the same project: Davis and Fill represents the view for the Institutional management point of view, another (Martin and Treves) represents the view of the departmental/subject leadership, and a third paper (Durham and Arrell) examines the challenges and benefits of cross institutional collaboration from faculty point of view. It is informative to observe the extent to which these viewpoints differ; the casual reader might not even notice that these papers discuss the same project.

While most of the projects are concerned with how the work of a funded project will be sustained after funding is completed, Cook et al. describe work on re-usable learning objects at London Metropolitan University that has matured into a funded project (The RLO-CETL). 
Finally White draws conclusions from research over six institutions from across the spectrum of UK Universities that concludes that the factors for successful embedding may depend on organisational and managerial structures of institutions.

The workshop finished with a round table discussion and summing up facilitated by Susan Eales. A number of critical success factors were identified and these can be grouped into 4 main areas

\section{Management and Organisational factors}

In order to effect cultural change to enable the sustained use of e-learning tools and techniques in higher education, there has to be top down management involvement in the form of new strategies and policies. However, in order for these to work, they must be implemented for clearly articulated reasons that are derived from a need to change, that put education before technology and are aligned with the mission and future aspirations of the institution. The strategies and policies require active involvement and commitment of senior management, not just a rubber stamp in a committee meeting. In this way, organisational objectives can be harnessed to make the change and the organisational structure utilised to nurture it.

It is not realistic to expect that adoption of e-learning tools and techniques will occur as part of the normal 'day job', management must be willing to invest in buyouts for staff on an ongoing basis as part of the strategy for sustainability.

\section{Collaboration and dissemination factors}

Awareness raising and dissemination internally is much more difficult than external dissemination as they require marketing skills and techniques that enable you to influence colleagues and change practice, rather than just giving information about the project. The use of enthusiastic faculty 'champions' to form communities of practice is a valuable way of beginning to change culture. Being honest by sharing good and bad practice, and also sharing models in the e-learning design process in order that people can recognise themselves within the models, can also be extremely useful factors in engaging the interest of colleagues. Embedding needs to be at all levels in the institution - technological, pedagogical and cultural - and support departments in the university, such as learner support services, can be valuable allies. Nevertheless, external visibility of projects does help enormously with internal recognition.

\section{3. e-Learning design factors}

The development and use of open standards and open source software is one of the most critical success factors in transforming e-learning from a 'cottage industry' to a sustainable, scalable way of offering learning opportunities that fit the aims and objectives of the $21^{\text {st }}$ Century higher education sector. However, other factors in the design process are also important for embedding. These include understanding disciplinary differences, ensuring that there is a whole curriculum approach and building learning objects with 
pedagogical pluralism to enable re-use and re-purposing. Those involved in X4L and Digital Libraries in the Classroom projects also found that the more people who share in the design process the more likely the result will have common sharable outcomes. Consideration should be given to the employing of students to bring a user and young person perspective to the design. Finally, agile learning technologist and technical support processes are needed in order to offer a 'just-in time' approach to support for the teacher in the classroom.

\title{
4. People factors
}

\begin{abstract}
Although the above factors all include people issues in an implicit way, it is worth adding here the other human factors that were highlighted in the workshop discussion as contributing towards culture change. Taking the time to fully understand the politic and culture of your institution and ensuring that changes made are compatible with these can enable you to identify and work with the resistance, rather than pushing against it. Engagement of the community in developing new strategies and policies will also give them a greater chance of success. Learning technologists who have a basic understanding of the subject area and of pedagogy; or a member of the team with both a technology and pedagogy background who can 'interpret' between academics and IT specialists are extremely valuable to the experience of elearning for both the teacher and student. Professional rewards and accreditation of innovation/developers/early adopters should be considered as recognition may encourage others to take part.
\end{abstract}

At the time of publication of this special edition, the DLIC projects are in the later stages of "embedding". It was a requirement of funding that the institutions involved in the projects funded the final 2 years of the 5-year programme themselves. It is the ultimate test of whether an innovation has been successful and embedded if there has been change which continues after the money ceases.

\section{The Editors}

Hugh Davis is the University Director of eLearning at the University of Southampton, and also Head of the Learning Technology Research Group within Electronics and Computer Science. He has been involved as a change agent within university education for many years; he is currently principal investigator on the DialogPLUS project, which has been cooperatively designing learning activities for use in undergraduate Geography teaching with partners in UCSB, Leeds and Penn State

universities

Susan Eales was a Programme Manager at JISC for three years where her responsibilities included managing the Digital Libraries in the Classroom and Exchange for Learning Programmes. She has an extensive overview of the issues emanating from the projects in those and other programmes, especially those to do with cultural change. Since June 2006, Susan has been Electronics Services Development Manager for the UK's Open University Library and Learning Resources Centre 
Acknowledgement: The editors would like to express their enormous gratitude to Karen Fill - whose efforts have made this edition possible. 